\title{
OPTICAL APERTURE SYNTHETIC IMAGES OF THE PHOTOSPHERE AND MOLECULAR ATMOSPHERE OF MIRA
}

\author{
C. A. Haniff, ${ }^{1}$ A. M. Ghez, P. W. Gorham, ${ }^{2}$ S. R. Kulkarni, K. Matthews, and G. Neugebauer \\ Palomar Observatory, California Institute of Technology, Pasadena, California 91125 \\ Received 7 November 1991; revised 21 January 1992
}

\begin{abstract}
We have used aperture synthetic imaging methods to obtain diffraction-limited images of the photosphere and molecular atmosphere of the long period variable star Mira ( $o$ Ceti). These maps, obtained close to the peak of Mira's variability cycle, clearly indicate substantial distortions from circular symmetry. Our image of the emission due to resonant scattering of TiO shows a significant contribution due to a cool atmosphere with a radius one and a half times as great as that of the photosphere. We suggest that the photospheric asymmetry is most likely associated with the intrinsic pulsation mode of the star and that stable nonradial pulsation modes can coexist with the radial modes that are responsible for the photometric variability. Further aperture synthetic mapping, which should be possible for a major fraction of the photometric cycle, is capable of verifying this hypothesis.
\end{abstract}

\section{INTRODUCTION}

The introduction of self-calibration (phase-closure) methods in optical astronomy (Haniff et al. 1987; Nakajima et al. 1989; Haniff 1989) now permits ground-based telescopes to be used as phase incoherent imaging interferometers. Unlike conventional speckle imaging techniques, these methods are characterized by the use of sparse telescope pupils obtained by masking off most of the clear aperture of the telescope with a nonredundant screen. Although limited in sensitivity when compared to fully filled aperture techniques, the nonredundant masking (NRM) approach is preferred for studies of bright sources because it provides images of higher dynamic range than would otherwise be attainable.

The initial tests of the NRM method have been mainly concerned with observations of binary stars (Haniff et al. 1987; Nakajima et al. 1989; Robertson et al. 1991), but the potential of the method for more complex sources has been most successfully demonstrated by the surface imaging of the M supergiant Betelgeuse (Buscher et al. 1990). These authors unambiguously identified a hotspot on the stellar surface contributing $\sim 10 \%$ of the total flux at visual wavelengths.

As part of a continuing program of high-resolution imaging at Palomar we have begun a multiepoch survey of nearby evolved stars and their circumstellar environments. In this paper we present our first epoch NRM images of the long period variable Mira. In the following section we briefly review the observations and the data reduction methods utilized to recover diffraction-limited images from the raw interferograms. Our results are described in Sec. 3 and discussed in Sec. 4. Conclusions are given in Sec. 5.

\footnotetext{
${ }^{1}$ Current address: Institute of Astronomy, Madingley Road, Cambridge, CB3 OHA, England and Mullard Radio Astronomy Observatory, Cavendish Laboratory, Madingley Road, Cambridge, CB3 OHE, England.

${ }^{2}$ Current address: Department of Physics and Astronomy, University of Hawaii at Manoa, 2505 Correa Rd., Honolulu, HI 96822.
}

\section{OBSERVATIONS AND DATA REDUCTION}

The observations reported here were made at the $f / 415$ Gregorian focus of the Hale $5 \mathrm{~m}$ telescope on the nights of 7-10 September 1990. In this configuration, which is different from the optical arrangement used for our first NRM experiments (Nakajima et al. 1989), a small concave secondary mirror located just beyond the $f / 3.3$ primary focus reflected starlight directly toward a resistive anode photoncounting camera giving a final focal ratio of $f / 415$. The telescope pupil was screened with a linear five-hole nonredundant mask (effective subaperture size $=15 \mathrm{~cm}$ ) which was located in the converging beam at the focus of the primary mirror, $150 \mathrm{~mm}$ in front of the Gregorian secondary. This provided ten baselines in the range $36-450 \mathrm{~cm}$. Because of the use of a one-dimensional mask, the mask was rotated with respect to the source in order to obtain two-dimensional coverage of the source visibility function. The sequence of rotations was performed in a quasirandom manner so as to minimize the possibility of mistaking any temporal trend in the visibility data for one related to the position angle of the source with respect to the nonredundant mask. Typically, 12 rotations of the mask were secured so as to achieve adequate UV coverage for image recovery.

Photon addresses from the camera were digitized to 9 bits which, for this optical configuration, gave an effective pixel scale of 4.9 marcsec and a field of view of 2.5". This small pixel scale, coupled with the use of approximately $r_{0}$-sized mask subapertures, ensured that deadtime effects in the detector were not a problem at the counting rates experienced $\left(\leqslant 40 \times 10^{3} \mathrm{~s}^{-1}\right)$. The observations of Mira were taken in narrow $(\sim 7.5 \mathrm{~nm})$ spectral channels in the "pseudocontinuum" at $700.7 \mathrm{~nm}$, in a deep $\mathrm{TiO}$ absorption band at 709.9 $\mathrm{nm}$, and at an intermediate region of the spectrum centered on $650.0 \mathrm{~nm}$, and were interleaved with observations of nearby unresolved calibration sources at $\sim 5 \mathrm{~min}$ intervals. Because of the small bandwidths and relatively long central wavelengths of the filters used, and the moderate air masses of the sources (1.2-1.3) no atmospheric dispersion corrector was utilized. At the time of the observations the variabil- 
ity phase of Mira was $\sim 0.94$. Further details of the observations are given in Table 1.

Reduction of the data followed standard nonredundant masking procedures (see e.g., Haniff et al. 1987; Nakajima et al. 1989) in which photon-bias-corrected estimates of the visibility amplitude squared and bispectrum were evaluated at the ten spatial frequencies and at the ten bispectrum coordinates defined by the pupil mask at each position angle. In order to increase the signal-to-noise ratio of the bispectral measurements, local averaging (Gorham et al. 1989) of the bispectrum was performed over the 12 nearest neighbors of each bispectrum element. Because our photon counting camera "time tags" each individual photon event the raw data were analyzed using a range of software integration times, the final mean integration times being chosen so as to maximize the signal-to-noise ratios of the accumulated power spectra and bispectra, respectively (see Table 1). Once averaged, both the source power spectra and bispectra were calibrated using the reference source data to give unbiased estimates of the sampled source visibility amplitude and bispectrum phase. This procedure compensates for the residual effects due to fixed telescope aberrations and the small amount of atmospheric dispersion ( $\sim 10$ marcsec) across the filter bandwidths, as well as removing the reduction in mean visibility due to the statistical effects of the atmosphere.

Subsequent analysis of these calibrated data followed two paths. First of all, simple source models, such as uniformly illuminated discs, were fit to the Fourier data to identify the overall structures present in the source. However, rather than fitting more complicated models directly, the visibility amplitudes and bispectrum phases were inverted using well established radio astronomical self-calibration methods (Pearson \& Readhead 1984) to obtain diffraction-limited images of Mira. In order to assess the possible influence of the reconstruction method on the recovered images all the restorations were carried out using both CLEAN and Maximum Entropy based algorithms. These gave images identical above the $10 \%$ contour level: at lower levels the images differed mainly in the smoothness of the weak diffuse components with, as expected, the emission in the MEM maps appearing less clumpy than that in the CLEANed counterparts. In addition, images were also made using only those visibilities corresponding to baselines shorter than $3.15 \mathrm{~m}$ so as to estimate the influence of the lowest signal-to-noise data on the recovered images.
3. RESULTS

In Fig. 1 we show the normalized visibility amplitude of Mira measured at $700.7 \mathrm{~nm}$ as a function of baseline length for two orientations of the pupil-plane mask, together with the predicted visibility function of the best-fitting uniformly illuminated circular disk model, which has a diameter of 51 marcsec [ $\equiv 920 R_{\odot}$ at 84 pc (Celis 1981)]. Although the fit is in qualitative agreement with the data, and consistent with previous speckle measurements made at the same wavelength and phase (Labeyrie et al. 1977; Bonneau et al. 1982) the data are of sufficient quality to rule out this circularly symmetric model. To further highlight the asymmetry we have plotted the visibility amplitude of Mira as a function of mask position angle for four of the ten baselines in Fig. 2 . The large modulation of the visibility amplitude provides a clear signature of asymmetric structure. Superimposed on Fig. 2 are the predicted visibility amplitudes for a uniform elliptical disk model that best fits the complete dataset at this wavelength: this has a major axis of $55 \pm 1$ marcsec (990 $R_{\odot}$ ), an axial ratio of $0.82 \pm 0.01$ and is oriented in position angle $119^{\circ} \pm 5^{\circ}$. Similar fits were performed using the data obtained at 709.9 and $650.0 \mathrm{~nm}$ - these results are summarized in Table 2.

However, it is evident from Fig. 2 that even the best-fit elliptical model agrees poorly with the data; for example, it systematically overestimates the visibility amplitudes at the shorter baselines and underestimates them at the longer baselines. As a result we caution the reader against taking the figures in Table 2 as accurate representations of the form of Mira, although they undoubtedly reflect gross trends in the stellar shape. In order to provide a more straightforward representation of our measurements, and rather than attempt to fit more complicated models, we have inverted the visibility data to obtain true images of the source. Figures 3(a)-3(c) [Plate 101] show the high-resolution CLEANed images of Mira at three different wavelengths. Contour representations of the images are given in Figs. 4(a)-4(c). These maps have angular resolutions of $\sim 30$ marcsec, i.e., some 30 times better than would have been achieved by conventional imaging.

In the pseudocontinuum at $700.7 \mathrm{~nm}$ our image [Fig. 3 (a) ] shows a roughly elliptical photosphere with the peak of emission displaced toward the SW of the "disk." The dynamic range of the image, i.e., the ratio of the peak bright-

TABLE 1. Observing log.

\begin{tabular}{|c|c|c|c|c|c|c|c|c|c|}
\hline UT Date & $\begin{array}{c}\lambda \\
(\mathrm{nm})\end{array}$ & $\begin{array}{c}\delta \lambda \\
(\mathrm{nm})\end{array}$ & $\begin{array}{l}\text { No. of } \\
\text { position } \\
\text { angles }\end{array}$ & $\begin{array}{c}\text { Resolution } \\
\text { (mas) }\end{array}$ & $\begin{array}{c}T_{\text {exp }} \text { power } \\
\text { spectrum }(\mathrm{ms})\end{array}$ & $\begin{array}{c}T_{\text {exp }} \\
\text { bispectrum } \\
\text { (ms) }\end{array}$ & $\begin{array}{l}\text { Photon } \\
\text { rate } \\
(\mathrm{kHz})\end{array}$ & $\begin{array}{l}\text { Calibrator } \\
\text { source }\end{array}$ & $\begin{array}{l}\text { Seeing } \\
\text { (arcsec) }\end{array}$ \\
\hline $\begin{array}{l}1990 \text { September } 7 \\
1990 \text { September } 8 \\
1990 \text { September } 9 \\
1990 \text { September } 10\end{array}$ & $\begin{array}{l}700.7 \\
700.7 \\
709.9 \\
650.0\end{array}$ & $\begin{array}{l}7.0 \\
7.0 \\
8.0 \\
7.4\end{array}$ & $\begin{array}{r}6 \\
12 \\
14 \\
14\end{array}$ & $\begin{array}{l}28 \\
28 \\
30 \\
25\end{array}$ & $\begin{array}{l}10 \\
10 \\
10 \\
10\end{array}$ & $\begin{array}{l}20 \\
10 \\
20 \\
10\end{array}$ & $\begin{array}{l}40 \\
40 \\
22 \\
29\end{array}$ & $\begin{array}{l}\text { BS 779 } \\
\text { BS } 804 \\
\text { BS 804 } \\
\text { BS } 804\end{array}$ & $\begin{array}{l}1.0 \\
0.9 \\
1.1 \\
0.9\end{array}$ \\
\hline
\end{tabular}

Notes to TABLE 1

The columns give in increasing order (1) UT date of observation, (2) central wavelength of the interference filter used, (3) FWHM of the narrowband filter, (4) number of position angles at which the nonredundant mask was located, (5) FWHM of the restoring beam used for the image reconstructions, (6) software integration time used for power spectrum accumulation, (7) software integration time used for bispectrum accumulation, (8) detected photon rate for Mira, (9) point source calibrator name, (10) long exposure seeing (FWHM). 


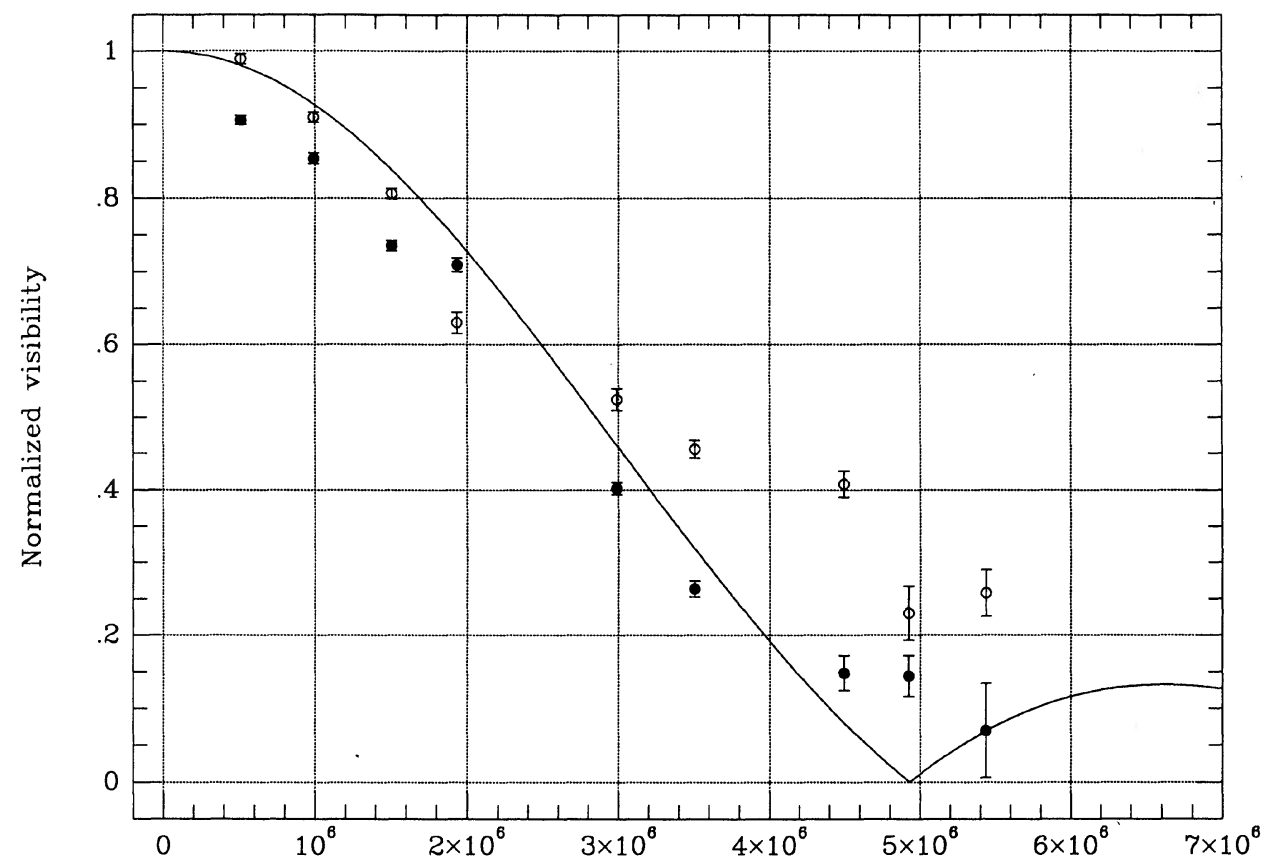

Projected baseline (lambda)
FIG. 1. Normalized visibility amplitudes of Mira measured at $700.7 \mathrm{~nm}$ on 1990 September 8 for two different nonredundant mask orientations: filled symbols $=$ P. A. $123^{\circ}$, open symbols $=$ P. A. $23^{\circ}$. At these two position angles the nonredundant mask was displaced slightly from its desired location and one of the subpupils was not fully illuminated. Consequently the longest baseline data have very low signal-tonoise ratios and have not been plotted. This was not a problem for the other position angles measured. $1 \sigma$ error bars are shown-these include the effects of photon noise but do not incorporate systematic uncertainties due to possible seeing fluctuations during the observations of source and calibrator. The solid curve shows the visibility function of the best-fitting uniformly illuminated disk model, which has a diameter of 51 marcsec $(920$ $\left.R_{\odot}\right)$. ness to the weakest believable feature in the map, is $33: 1$ based on the consistency of the maps made using differing inversion methods and different sets of data. In the nearby TiO band at $709.9 \mathrm{~nm}$ [Fig. 3(b) ] the image is considerably larger - the best fitting elliptical disk model has a major axis of 80 marcsec $\left(1450 R_{\odot}\right)$-and comprises a bright elliptical core with roughly the same orientation as the $700.7 \mathrm{~nm}$ photospheric image together with a more symmetrically disposed halo of emission. Because of the depth of this molecular absorption feature and the fact that the source is significantly more resolved than in the nearby pseudocontinuum the dynamic range of the map is somewhat lower at 25:1. At $650.0 \mathrm{~nm}$ [Fig. 3(c)] the core asymmetry is again detected unambiguously and there is evidence for weaker extended emission to the north and south. The data are consistent with a slightly larger extent than that at $700.7 \mathrm{~nm}$, with an elliptical disk fit giving a major axis of 60 marcsec $\left(1080 R_{\odot}\right.$ ) aligned in P.A. $108^{\circ}$. The dynamic range of the map is $\sim 25: 1$.

To further highlight the photospheric extension seen in the $\mathrm{TiO}$ band we have subtracted a scaled version of the $700.7 \mathrm{~nm}$ continuum image from the $709.9 \mathrm{~nm}$ map. Because the reconstructed images have been recovered using selfcalibration methods, the absolute positional information of the source is lost, and so we have used the peak of the stellar flux to register the two images. Similarly, an absolute scaling factor for the continuum image cannot be derived from our data so the resulting map, shown in Fig. 3(d), is useful mainly in revealing the spatial details of the cooler TiO emitting layers. The halo emission, which arises primarily because of resonant scattering, is somewhat more symmetrically disposed than the photospheric core, but interestingly appears weakest perpendicular to the photospheric extension.

\section{DISCUSSION}

The primary results of this study have been the detection of substantial departures from spherical symmetry in the brightness distribution of Mira. Such features were initially detected directly at optical wavelengths by Karovska et al. (1991) who secured the first speckle observations of sufficient quality to reveal them. Although the position angle of the asymmetry seen at $700.7 \mathrm{~nm}$ agrees well with two independent measurements made at similar phases (Karovska $e t$ al. 1991; Wilson et al., 1992) the interpretation of our data in terms of a simple uniformly illuminated elliptical disk model is far from satisfactory. In particular, none of the diffractionlimited images show surface brightness distributions that have their peaks centered within the envelope defined by the outermost contour levels.

The most understandable features of the maps are the variations in apparent size of Mira as a function of wavelength. Because of the complex nature of the optical spectra of late type stars such as Mira (for which the "true continuum" is contaminated by numerous molecular absorption bands) it is possible to identify the wavelength ranges used for these observations with three physically separate layers in the stellar atmosphere (see e.g., Scholz \& Takeda 1987). Both the 650.0 and $709.9 \mathrm{~nm}$ bands are affected by absorption features, the former by an atomic line blend at $649.7 \mathrm{~nm}$, and the latter by a deep molecular TiO band. On the other hand, at $700.7 \mathrm{~nm}$ the spectral energy distribution of Mira is relatively free from such features and so probes the deeper "photospheric" layers of the atmosphere. Recent model atmosphere calculations (Bessell et al. 1989) predict the apparent diameters of Mira at 650, 700, and $710 \mathrm{~nm}$ to be in the ratio $1.10: 1.00: 1.32$, which is in reasonable agreement with the 

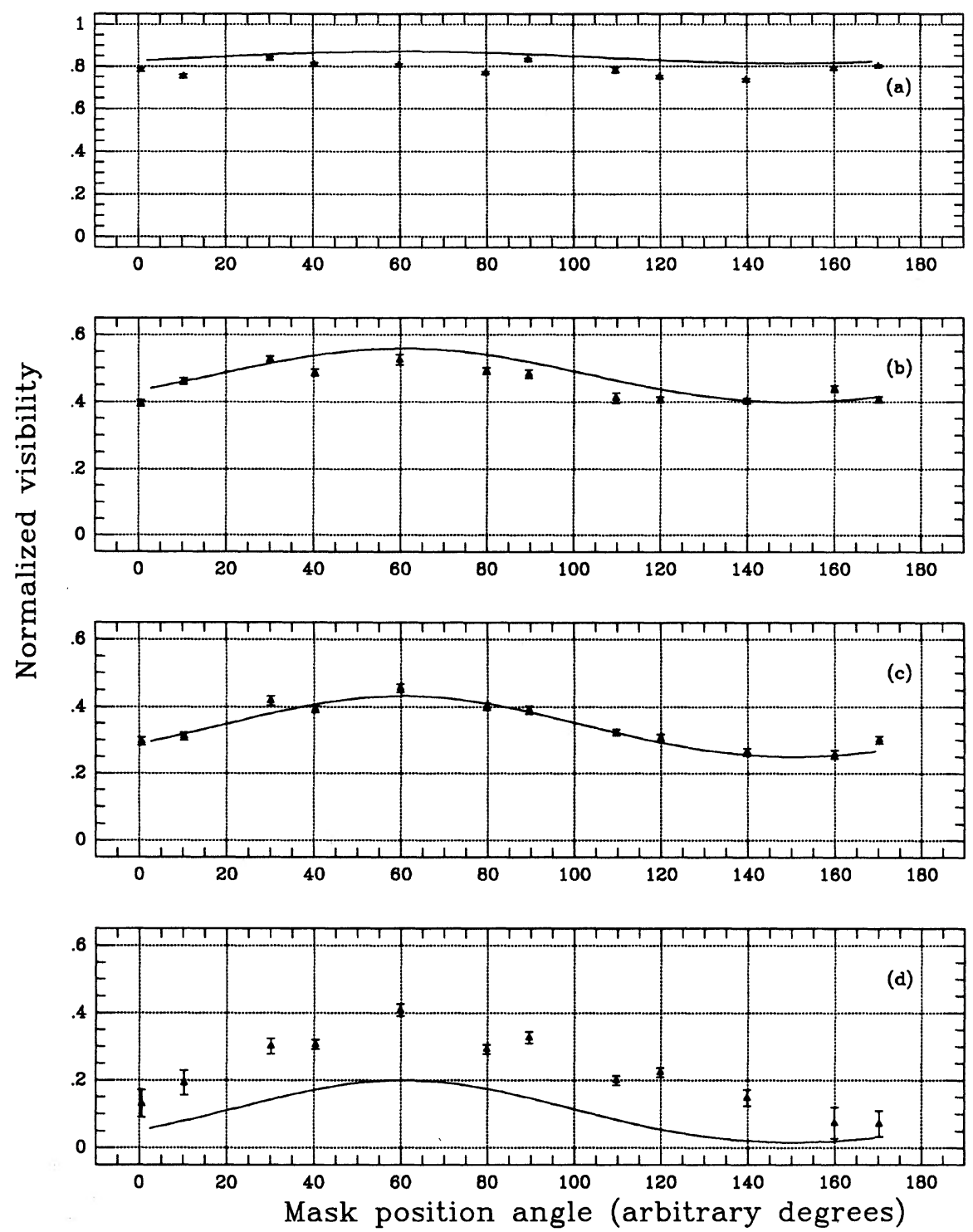

FIG. 2. Normalized visibility amplitude of Mira as a function of mask position angle at $700.7 \mathrm{~nm}$. The different panels correspond to baselines of (a) $1.06 \mathrm{~m}$, (b) $2.10 \mathrm{~m}$, (c) $2.46 \mathrm{~m}$, and (d) $3.15 \mathrm{~m}$, respectively. Overplotted on each panel is the visibility amplitude of the uniform elliptical disk model that best fits the complete set of Fourier data obtained at this wavelength.

TABLE 2. Model-fitting results.

\begin{tabular}{cccccc}
\hline \hline UT Date & $\begin{array}{c}\lambda \\
(\mathrm{nm})\end{array}$ & $\begin{array}{c}\text { Major axis } \\
(\mathrm{mas})\end{array}$ & $\begin{array}{c}\text { Major axis } \\
\left(R_{\odot}\right)\end{array}$ & Axial ratio & Position angle \\
\hline 1990 September 7 & 700.7 & $52 \pm 2$ & 940 & $0.78 \pm 0.02$ & $105^{\circ} \pm 10^{\circ}$ \\
1990 September 8 & 700.7 & $55 \pm 1$ & 990 & $0.82 \pm 0.01$ & $119^{\circ} \pm 5^{\circ}$ \\
1990 September 9 & 709.9 & $80 \pm 1$ & 1450 & $0.85 \pm 0.02$ & $158^{\circ} \pm 10^{\circ}$ \\
1990 September 10 & 650.0 & $60 \pm 1$ & 1080 & $0.85 \pm 0.02$ & $108^{\circ} \pm 5^{\circ}$ \\
\hline \hline
\end{tabular}

Notes to TABLE 2

Uniform elliptical disk parameters obtained from model fitting. The columns give in increasing order (1) UT date of observation, (2) central wavelength of the interference filter used, (3) major axis of the best-fitting uniform elliptical disk, (4) as for column 3 but computed using an assumed distance to Mira of 84 pc, (5) axial ratio of the best-fitting uniform elliptical disk, (6) position angle of the major axis of the best-fitting uniform elliptical disk. 

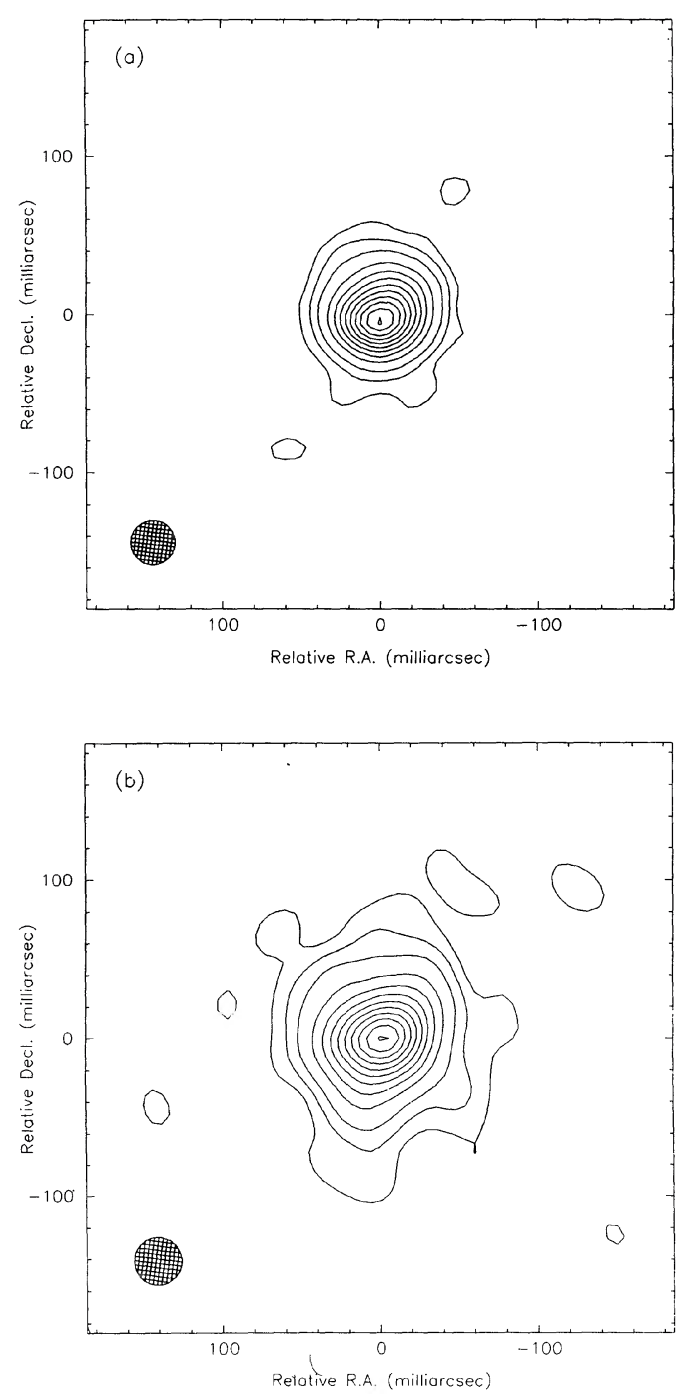

observed ratio of 1.09:1.00:1.45 despite the fact that these models assume spherically symmetric atmospheres.

The asymmetries present at all three wavelengths are more difficult to understand although for some time now their presence had been inferred by, for example, polarimetry (Shawl 1975; Boyle et al. 1986), or more directly, by CO observations (Planesas et al. 1990). At least three separate causes can be envisaged: (i) large-scale temperature fluctuations on the stellar surface due to, e.g., giant convective cells, (ii) a shape asymmetry related to stellar pulsation, and (iii) perturbations in the equilibrium form of the star due to interaction with a companion. Although Mira has a secondary companion (Baize 1980), the 400 yr orbital period of the companion renders the latter of these possibilities most unlikely. The presence of large scale convective features or hot spots is a more attractive explanation, but the scale size of the asymmetry we detect, i.e., a substantial fraction of the stellar disk, is at the limit of the possible range of sizes allowed in theoretical scenarios (Schwarzschild 1975). Furthermore, the expected visual brightness fluctuations of

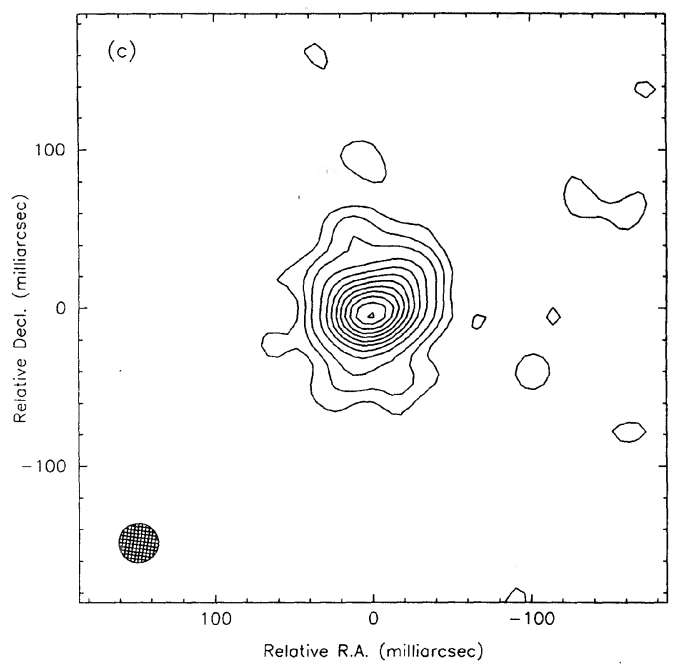

Fig. 4. Contour representations of the diffraction-limited images of Mira at (a) $700.7 \mathrm{~nm}$, (b) $709.9 \mathrm{~nm}$, and (c) $650.0 \mathrm{~nm}$. The contour levels have been plotted at $2 \%, 5 \%, 10 \%, 20 \%$, $30 \%, 40 \%, 50 \%, 60 \%, 70 \%, 80 \%, 90 \%$, and $99 \%$ of the peak intensity. The circle in the lower left-hand corner of each image shows the restoring beam. The orientation of the images is the same as in Fig. 3. a single such cell would exceed a factor of 10 , and so it is unlikely that such perturbations to the regular light curve of Mira would have gone unnoticed. In addition, the recent discovery of temporal variations in the asymmetry over timescales of a few months (Quirrenbach 1991) argues against this model which predicts a considerably longer evolutionary timescale.

Perhaps the most favored possibility is that we are seeing an effect related to the $\mathbf{3 3 2}$ day period pulsation of the star. The observed alignment of the overall elliptical symmetry of the photosphere is in agreement with the results of Karovska et al. (1991) who were able to fit elliptical disk models with major-axis position angles of $115^{\circ} \pm 10^{\circ}$ and $135^{\circ} \pm 10^{\circ}$ to power spectra of Mira measured at phase 0.97 in 1987 and 1988. The detection of this consistent axis for the photospheric asymmetry over 4 variability maxima, is strongly suggestive of a causal link between the pulsation of the star and the apparent photospheric distortion. If this conjecture is correct then it would seem that relatively stable nonradial modes can exist in giant stars together with the radial modes 
responsible for their photometric variability. In this case though, there would be no need for the periods of the two modes to be commensurable and so it may be fortuitous that the position angles and phases of the observed asymmetry agree. However, the strong correlation of polarization with variability phase (Boyle et al. 1986) argues against this hypothesis.

Further progress in our understanding awaits a systematic study of the figure of Mira as a function of pulsation phase, both to characterize the brighter elliptical core asymmetry and the diffuse asymmetric features seen in the cooler atmospheric layers. Recent work (Haniff \& Buscher 1991) has shown that it is possible to extend the nonredundant masking method to fainter magnitudes while maintaining a high dynamic range, so that reliable diffraction-limited imaging of Mira over the whole of its variability cycle should soon be possible. In particular these authors show that even at its photometric minimum, the use of a pupil plane mask is likely to be advantageous when compared to the use of a filled aperture if visibility data beyond the first maximum are required.

\section{CONCLUSIONS}

We have presented diffraction-limited optical aperture synthesis images of the nearby long period variable Mira at three wavelengths. Though agreeing in overall extent with the predictions of spherically symmetric model atmosphere calculations, they confirm the results of Karovska et al. (1991) in detecting gross deviations from spherical symmetry. The consistency of the axis of the asymmetry at three variability maxima, together with its recently reported rapid variability, suggests an origin related to the pulsation of the star. Time-resolved mapping at these high spatial resolutions should be able to confirm this hypothesis.

We are grateful to E. S. Phinney and N. Murray for useful discussions and to $\mathrm{H}$. Spinrad for the loan of the narrowband filters. Vital assistance at the telescope was provided by Juan Carrasco. S.R.K.'s research is funded in part by the Packard Foundation and a NSF Presidential Young Investigator award. C.A.H. was supported by a SERC/NATO research fellowship.

\section{REFERENCES}

Baize, P. 1980, A\&AS, 39, 83

Bessell, M. S., Brett, J. M. Scholz, M., \& Wood, P. R. 1989, A\&A, 213, 209

Bonneau, D., Foy, R., Blazit, A., \& Labeyrie, A. 1982, A\&A, 106, 235

Boyle, R. P., Aspin, C., Coyne, G. V., \& McLean, I. S. 1986, A\&A, 164, 310

Buscher, D. F., Haniff, C. A., Baldwin, J. E., \& Warner, P. J. 1990, MNRAS, 245, 7P

Celis, L. S. 1981, A\&A, 99, 58

Gorham, P. W., Ghez, A. M., Kulkarni, S. R. Nakajima, T., Neugebauer, G., Oke, J. B., \& Prince, T. A. 1989, AJ, 98, 1783

Haniff, C. A., Mackay, C. D., Titterington, D. J., Sivia, D., Baldwin, J. E., \& Warner, P. J. 1987, Nature, 328, 694

Haniff, C. A. 1989, in Diffraction-Limited Imaging with Very Large Telescopes, edited by J-M. Mariotti and D. Alloin, NATO ASI Series C (Kluwer, Dordrecht), pp. 171-190

Haniff, C. A., \& Buscher, D. F. 1991, in High-Resolution Imaging by Interferometry II, edited by F. Merkle (European Southern Observatory, Garching bei Munchen) (in press)

Karovska, M., Nisenson, P., Papaliolios, C., \& Boyle, R. P. 1991, ApJ, 374, L51
Labeyrie, A., Koechlin, L., Bonneau, D., Blazit, A., \& Foy, R. 1977, ApJ, 218, L75

Nakajima, T., Kulkarni, S. R., Gorham, P. W., Ghez, A. M., Neugebauer, G., Oke, J. B., Prince, T. A., \& Readhead, A.C.S. 1989, AJ, 97, 1510

Pearson, T. J., \& Readhead, A.C.S. 1984, ARA\&A, 22, 97

Planesas, P., Kenney, J., \& Bachiller, R. 1990, ApJ, 364, L9

Quirrenbach, A. 1991, in High-Resolution Imaging by Interferometry II, edited by F. Merkle (European Southern Observatory, Garching bei Munchen) (in press)

Robertson, J. G., Bedding, T. R., Marson, R. G., Gillingham, P. R., Frater, R. H., O'Sullivan, J. D., \& Norris, R. P. 1991, PASA, 9, 162

Shawl, S. J. 1975, AJ, 80, 602

Scholz, M., \& Takeda, Y. 1987, A\&A, 186, 200

Schwarzschild, M. 1975, ApJ, 195, 137

Wilson, R. W., Baldwin, J. E., Buscher, D. F., \& Warner, P. J. 1992, MNRAS (in press) 


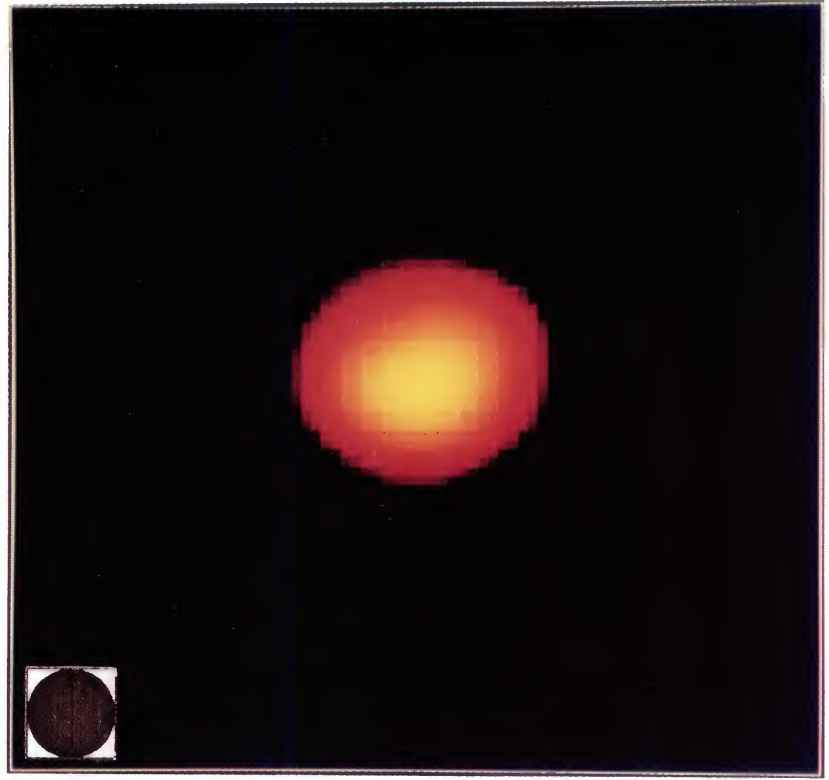

(a)

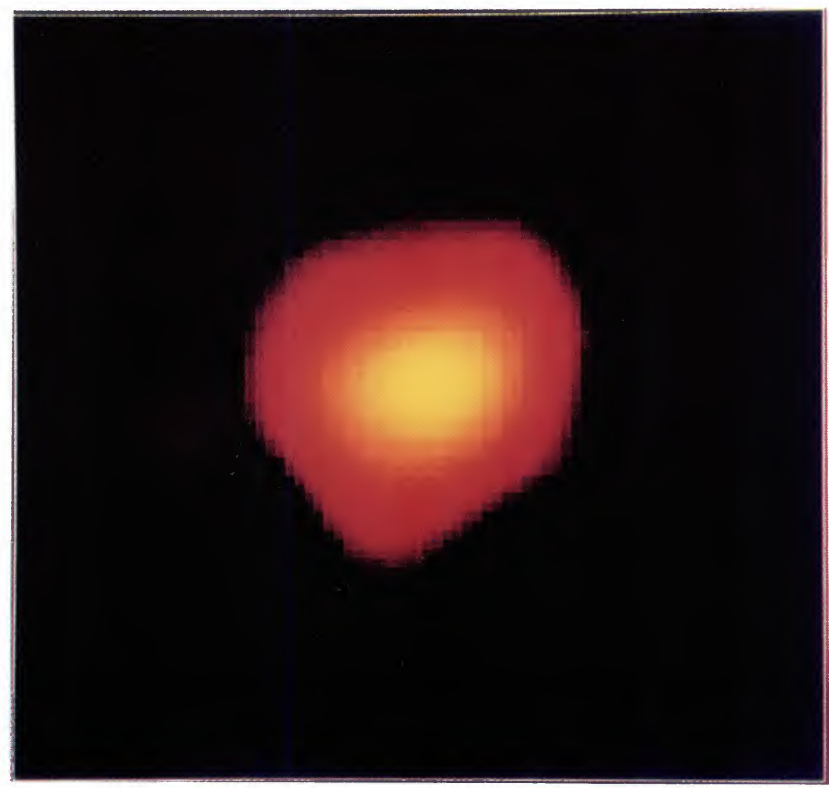

(b)

FIG. 3. Diffraction-limited images of Mira obtained at (a) $700.7 \mathrm{~nm}$, (b) $709.9 \mathrm{~nm}$, and (c) $650.0 \mathrm{~nm}$. Each image covers a field of $0.25^{\prime \prime} \times 0.25 "$ and is oriented with north to the top and east to the left. The circle in the lower left of panel (a) shows the restoring beam which has a FWHM of 28 mas. The beams for panels (b) and (c) are similar circular Gaussians with FWHM of 30 mas and 25 mas, respectively. The image in panel (d) has been obtained by subtracting a scaled version of the $700.7 \mathrm{~nm}$ map from that obtained at $709.9 \mathrm{~nm}$. This better reveals the large extent of the cooler TiO emitting shell that dominates the emission in the broad TiO band selected by the $709.9 \mathrm{~nm}$ filter. The dynamic ranges of the maps, here defined as the ratio of the peak brightness to the brightness of the weakest believable feature in the maps, are 33:1, 25:1, and 20:1 for panels (a), (b), and (c), respectively. The weak fingers to the north and south seen in the 709.9 and $650.0 \mathrm{~nm}$ maps are detected at $>$ the $3 \sigma$ level in both the MEM and CLEAN reconstructions, but their precise morphology is sensitive to the details of the inversion procedure. At the limiting sensitivity of all of the images there is no evidence for any extended emission beyond $\sim 80$ mas from the center of the star.

Haniff et al. (see page 1663)

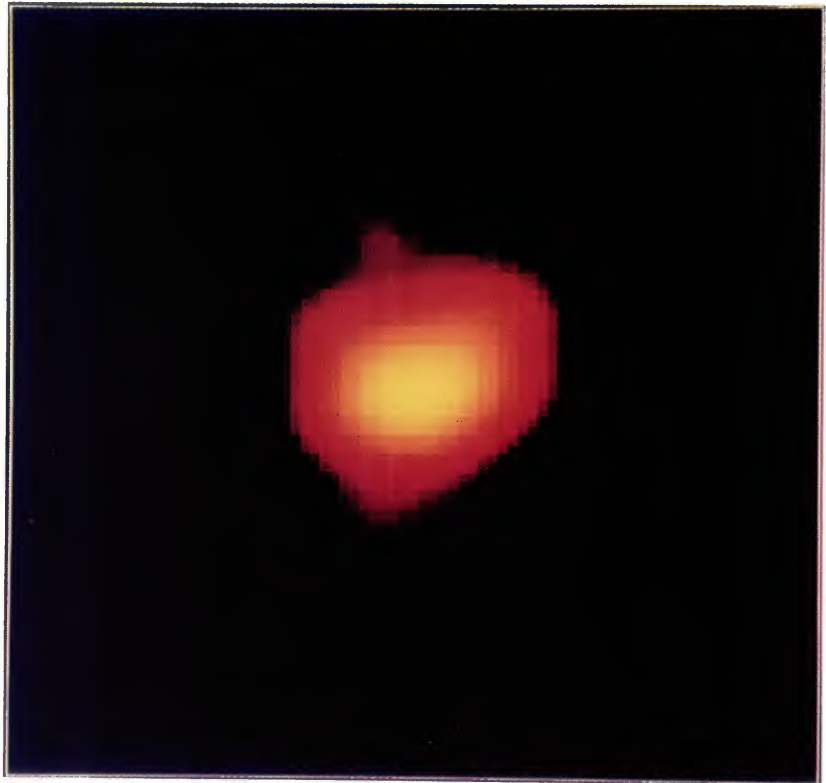

(c)

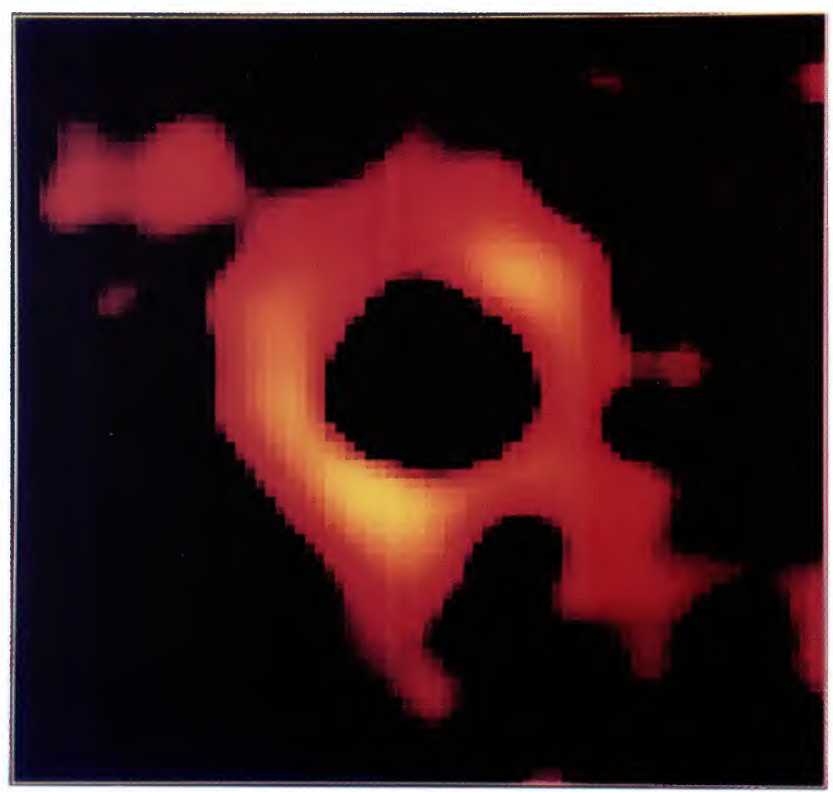

(d) 